\title{
Behavior of a Flat Solid in a Container with Liquid Subject to Large Amplitude Vibration
}

\author{
Victor Kozlov and Olga Vlasova \\ Laboratory of Vibrational Hydromechanics, Perm State Humanitarian Pedagogical University, 24 Sibirskaya Street, \\ Perm 614990, Russia \\ Correspondence should be addressed to Victor Kozlov; kozlov@pspu.ru
}

Received 5 May 2016; Accepted 31 August 2016

Academic Editor: Sergio De Rosa

Copyright ( 2016 V. Kozlov and O. Vlasova. This is an open access article distributed under the Creative Commons Attribution License, which permits unrestricted use, distribution, and reproduction in any medium, provided the original work is properly cited.

\begin{abstract}
We experimentally study the behavior of a flat body in the oscillating container with liquid. The body density is much more than the density of liquid. The body oscillates with large amplitude under the action of inertia forces. It is found that under the vibration the body lifts up and goes to quasi-steady suspended state at some distance from the container bottom. The lift force is measured by a method of dynamic suspension of a body in the gravity field. It is found that the dimensionless repulsion lift force depends on the dimensionless amplitude of the body oscillations; it reduces with the amplitude and is in agreement with the theoretical model in the limit of small amplitude. Qualitatively new regimes of body behavior are found in the supercritical region. With an increase of the vibration intensity, the body gets a stable state in the middle of the container height and then moves on to the container ceiling.
\end{abstract}

\section{Introduction}

Behavior of a solid inclusion in the oscillating container filled with liquid is an important technological task because both the vibrations and the inhomogeneity in density multiphase systems are widely distributed in modern technologies.

Let us consider the case of high frequencies when the thickness of the dynamic boundary layers is small compared to the inclusions size. In this case, the time-averaged lift forces acting on the oscillating body in liquid appear because of the asymmetric oscillating flow around the body, hence the asymmetric distribution of an average pressure upon its surface. A number of problems with oscillations of spherical and cylindrical bodies near the solid boundaries were theoretically considered in high-frequency approximation when the viscosity does not play role during the body oscillations $[1,2]$. It has been found that the body, which oscillates at some distance from the solid boundary, is attracted to it. The attractive force acts in both cases of longitudinal and transversal body oscillations relative to the boundary. In gravity field, the attractive force is able to balance the body weight and to keep the body near the container ceiling.
In case when the body oscillates at short distance from the wall, comparable with the thickness of viscous boundary layer, the inviscid oscillating flow around the body exists only from the outer side in relation to the wall. In this case, the repulsion force manifests itself, but not the attractive one. The repulsion force was first found and investigated in experiments with spherical body [3]. It was demonstrated that, with increase of a distance between the oscillating body and the wall, the repulsion force decreases, and at a distance more than a thickness of Stokes boundary layer the attractive force replaces the repulsion one. The lift force was measured by method of vibrational suspension of the body in the gravity field. The body has to oscillate with a significant amplitude in order to provide the suspension in the gravity field. In experiment, the cavity vibrations tangentially to the solid boundary provide this condition. For cylindrical bodies the vibrational lift force was studied using the same method in [4]. Because of high importance for microgravity conditions, the vibrational lift force, which acts on oscillating solid, attracts attention of scientists, which prove the existence of attraction force at relatively large distance $[5,6]$ as well as reporting the existence of repulsion force, which manifests 
itself with increase of liquid viscosity [7, 8]. Vibrational dynamics of spherical solids in mentioned works was studied mainly by direct numerical simulation; the list of articles could be found in [8]. The investigated dynamics of spherical inclusion qualitatively agrees with the theoretical model, elaborated for 2D (cylindrical) body [4].

The forces acting on the flat body in rectangular container which performs longitudinal oscillations in its plane have been considered in [9]. The body differs in density from the liquid and makes the longitudinal oscillations under the action of inertia forces. In this case, if the container is oriented horizontally, the heavy body repulses from the container bottom and light one goes away from the container ceiling. The theoretical description of this force was done in approximation of small amplitude and high frequency of vibration. It means that the thickness of viscous boundary layer is small compared to the body and the cavity sizes. The conditions of the body steady suspended state versus the relative body density and it relative thickness was found theoretically. Theoretical predictions of the threshold repulsion of a heavy body from the bottom and the light one, from the ceiling, were qualitatively confirmed in experiments with both light and heavy bodies. In experiments, the relative body density varied in range from 0.05 to 2.1. It was found that the bodies repulse at a distance comparable with the Stokes layer thickness. In contradistinction to the theory, the bodies in form of parallelepiped of finite length were used in experiments [9]. The body oscillations in suspended state often had irregular character.

The purpose of this paper is to study vibrational dynamics of flat bodies by far exceeding the surrounding liquid density in case of large amplitude oscillations in the cavity frame. It is shown that the vibrations provide swimming of bodies even if they are made of bronze. The qualitative difference of the average body dynamics from the theoretical prediction in case of high oscillation amplitude is found.

\section{The Methodology and Experimental Setup}

We experimentally investigate the influence of vibration on the heavy flat solid in rectangular container filled with liquid. Container 1 is made of Plexiglas in a form of a parallelepiped with the length $L=22.0 \mathrm{~cm}$, width $D=3.4 \mathrm{~cm}$, and height $H=2.0 \mathrm{~cm}$ (Figure 1). Body 2 is made of bronze in the form of a plate with length $l=8.5 \mathrm{~cm}$, width $a=3.1 \mathrm{~cm}$, and height $h=0.89 \mathrm{~cm}$; the radius of curvature of body edges is $r=$ $0.45 \mathrm{~cm}$. The relative body thickness is $\xi=h / h_{L}=0.77$, and here $h_{L}=H-h_{S}$ is the thickness of liquid layer above the body. The bodies of the same shape and size but of different densities $\rho_{S}=3.7-7.7 \mathrm{~g} / \mathrm{cm}^{3}$ are used in experiments.

We changed the density by removing the internal parts of the body; the thickness of the remaining wall is constant all over the body perimeter and equals to 2 or $3 \mathrm{~mm}$. We sealed the end faces of the bodies with the lavsan membrane. The water-glycerol solution was a working fluid; its kinematic viscosity $v$ varies in the range $0.11-1.21$ St by changing the mass fraction of glycerol in the solution. Herewith the liquid density changes in the interval $\rho_{L}=1.18-1.23 \mathrm{~g} / \mathrm{cm}^{3}$, and the relative body density varies in the range $\rho=\rho_{S} / \rho_{L}=$
3.0-6.5. In some experiments, the aluminum powder is added for visualization of liquid motion inside the cavity. The mass fraction of the powder in the solution does not exceed $1 \%$.

Note that the elastic bumpers are attached to the inner ends of the container (dedicated area on Figure 2). They prevent the body blows with the cavity ends. After exclusion of the elastic bumpers the operating container length is $19 \mathrm{~cm}$, which corresponds to $L / h_{S}=21.1$.

The container with liquid and solid is fastened to the table of the mechanical vibrator which performs the horizontal translational oscillations according to the law $X=b \cos \Omega t$. The description of vibrator is available in [9]. The oscillations frequency $f=\Omega / 2 \pi$ varies from 1 to $25 \mathrm{~Hz}$, and the vibration amplitude $b$ varies in the range $1-5 \mathrm{~cm}$. The amplitude of vibrations is measured according the track length of the reflective label on the front wall of the container with an accuracy $0.1 \mathrm{~mm}$. The photos of a vibrating cavity are captured by high resolution camera. The control of vibration frequency is performed using the digital tachometer TD-3M with an accuracy $0.1 \mathrm{~Hz}$.

An observation of the body behavior is carried out through the front end face of the cavity. The body dynamics is studied using the photo-video recording (Figure 2). During the experiment the container is illuminated by the constant light source for monitoring the body behavior; the stroboscopic illumination is used to study the body dynamics in a certain phase of the oscillation. The camera (camcorder) is focused on the front body wall. The photo registration with an exposure time much less than the period of the container oscillations and high-speed video are used in monitoring the behavior of the body. High-frequency video recording is done by camera CamRecord 120 CL600x2 at frame rate $500 \mathrm{fps}$ and resolution $1280 \times 1024$ pixels per frame. Processing of the photo and video information is performed by specialized applications on the computer.

During the processing the coordinates $x$ of body position in a reference system of the container are measured; the positive $x$ corresponds to the body displacement to right (Figure 1). The distance to the lower boundary of the container is measured for each of the body edges. The positive value of the gap $d$ means vertical body lift. The angle of the body slope $\alpha$ is determined as $\alpha=\arctan \left(\left(d^{\text {right }}-\right.\right.$ $\left.d^{\text {left }}\right) / l$ ), and the positive value of an angle corresponds to the counterclockwise tilt of the body.

\section{Experimental Results}

3.1. Solid Body Vibrational Suspension. In the absence of vibration, the heavy body rests at the bottom of the container. The heavy solid made of bronze under the action of horizontal vibrations makes the longitudinal vibrations with respect to the cavity, remaining at the bottom. The increase of frequency $f$ (at definite amplitude $b$ ) brings to the threshold transition of the body to the suspended state (Figure 3). A similar effect was observed in experiments with the bodies of lower density [9]. The body position in the center along the container length is unstable. After transition of the body to the suspended state, it moves towards one of the container ends. The direction of movement to the left or to the right 


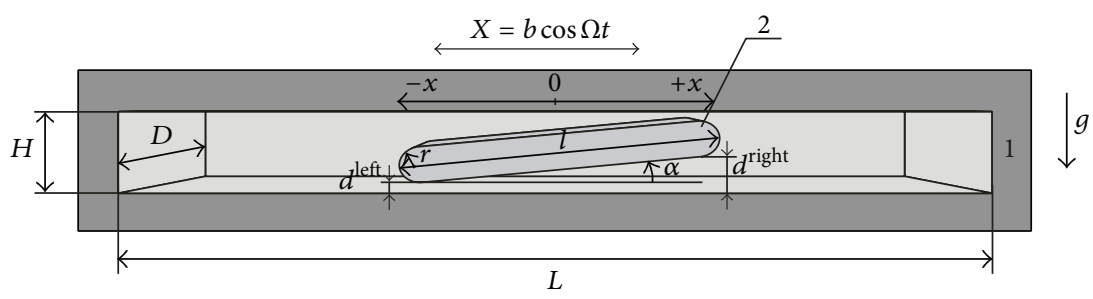

FIGURE 1: The container with a flat body placed in it.

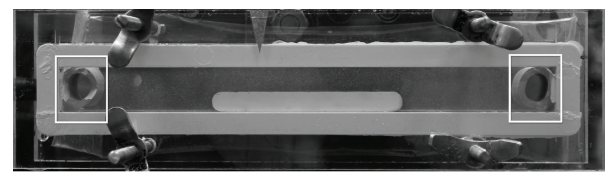

Figure 2: Photo of the container with a body fastened on a vibrator.

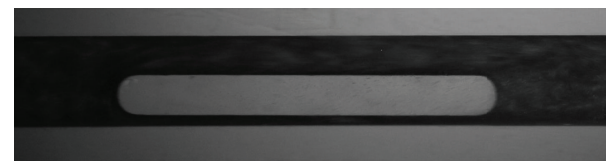

FIgURE 3: The quasi-equilibrium suspended state of the body in a vibrating cavity, $\rho=6.5, v=1.06 \mathrm{St}, f=6.4 \mathrm{~Hz}, b=36.6 \mathrm{~mm}$.

container ends takes place in occasional way. With decrease of vibrations intensity, the body returns to the bottom.

With increase of the vibration amplitude $b$, the critical frequency $f$ of the body separation from the cavity bottom decreases (Figure 4). All transitions occur in a threshold manner and with hysteresis. It is found that the liquid viscosity does not affect the value of the threshold frequency for the body of definite relative density. One can see in the plot that the experimental data of the threshold transitions in case of the body with relative density $\rho=6.4$ in liquid of different viscosity (from 0.12 to $1.21 \mathrm{St}$ ) are in satisfactory agreement with each other. The solid symbols denote the threshold repulsion of the body and the empty ones the threshold of body return to the boundary. The hysteresis area between the transitions is shown by hatching. The threshold transitions in case of bodies of different density have a similar shape.

With increase of the relative body density the thresholds (the critical value $f$ at definite $b$ ) of the repulsion and the return of the body increase, and the hysteresis area expands.

\section{Behavior of Heavy Body Depending on the Relative Density}

4.1. Heavy Body, $\rho=6.49$. The character of a flat body oscillation relative to the container in liquid of viscosity $v=0.13 \mathrm{St}$ is studied with the use of high-speed video camera. The container and the body oscillations along the horizontal axis are shown in Figure 5: (a): $f=7.1 \mathrm{~Hz}$ (before repulsion, $d=0$ ), (b): $f=12.7 \mathrm{~Hz}$ (in suspended state, $d \neq 0$ ). Hereinafter the solid symbols $x$ in the plot represent the position of the body relative to the container and open

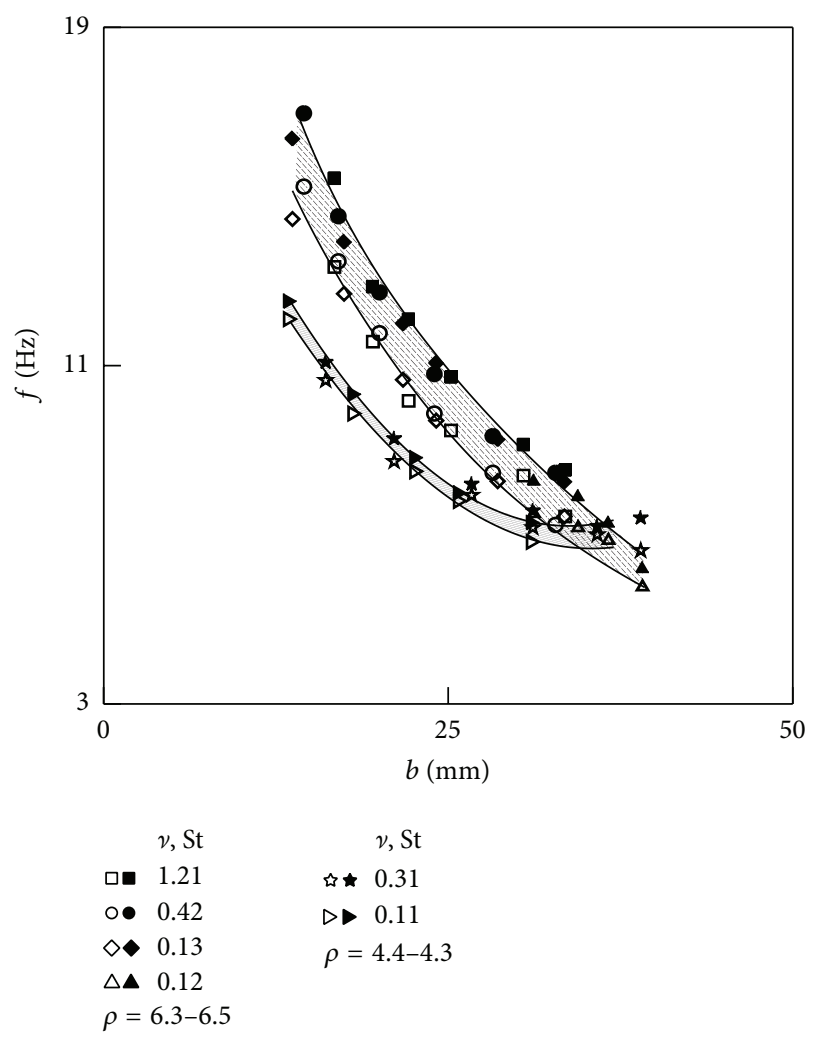

FIgURE 4: The boundaries of transitions in the suspended state for the bodies of different relative density in the fluids of different viscosity.

symbols $X$ the position of the container in the laboratory frame. One can see that before the repulsion the body oscillates in antiphase with the container oscillations but with some lag (Figure 5(a)). In a suspended state, the oscillations of the body and the container occur strictly in opposite phases (Figure 5(b)). Note that after the body transition to the suspended state the amplitude of its oscillations slightly increases.

In addition to longitudinal oscillations the body performs the angular oscillations of small amplitude; the amplitude does not exceed 0.5 degrees (Figure 5(c)). The angular oscillations are close to harmonic ones. The change of the sign of the angle $\alpha$ over time shows that the right and the left edges of the body rise periodically. The variation of the gap between the right body edge and container boundary over time is shown in Figure 5(d), and the same for the left edge in 


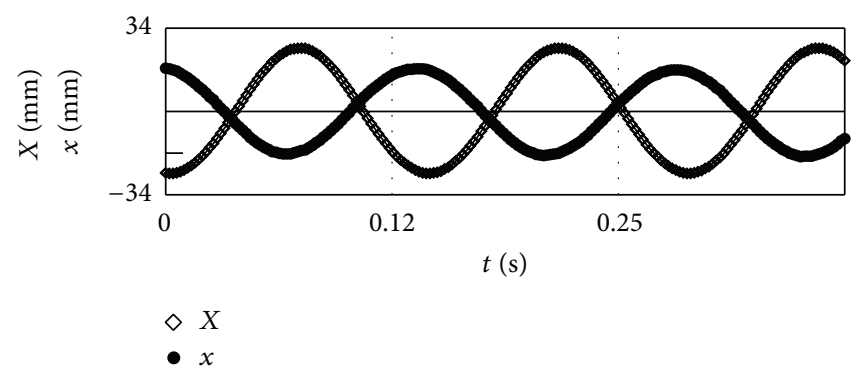

(a)

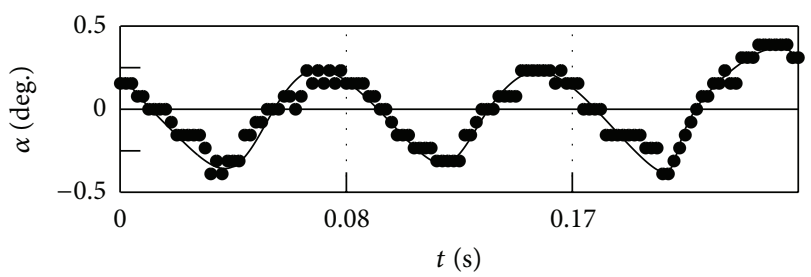

(c)

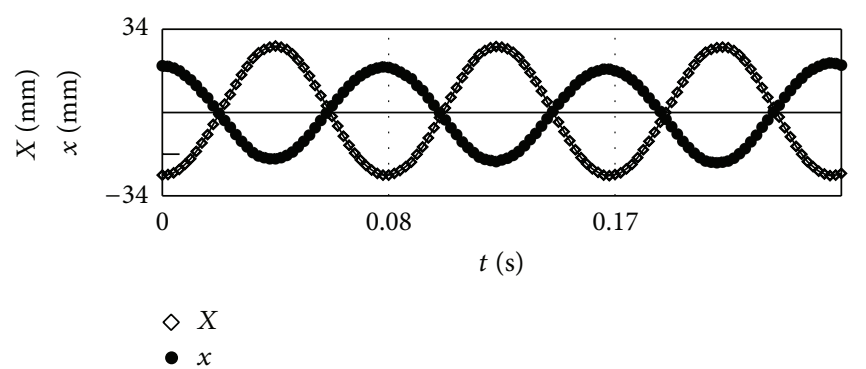

(b)

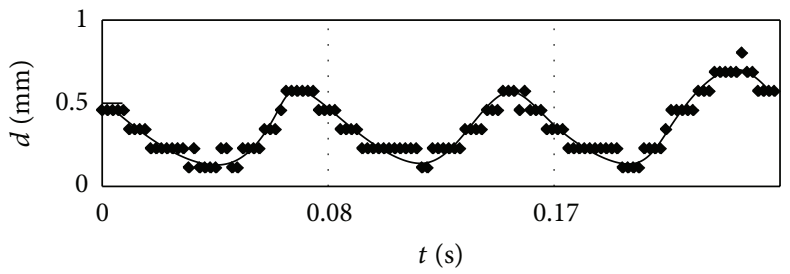

(d)

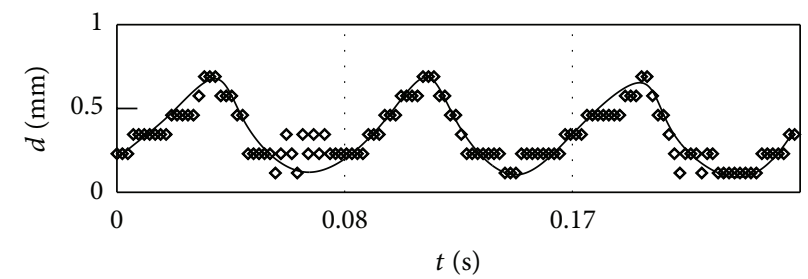

(e)

FIGURE 5: Characteristics of the body oscillations before (a) and after repulsion from the bottom (b-e).

Figure 5(e). During the angular oscillations, at the moment when one of the body edges is maximally remoted from the container boundary, another edge is in close proximity to the boundary but does not touch it.

The photos of the container during the vibrations at the extreme right (a) and the extreme left (b) positions are shown in Figure 6. As previously mentioned, the oscillating body moves to one of the container ends. In this experiment, the body performs oscillations near the left container end. Note that the body edge, which is closer to the container end, rises a little higher than the opposite one during the angular oscillations. This feature of the asymmetrical angular oscillations near the lateral container ends is typical for the flat bodies, both light and heavy [9].

The angle of the body slope $\alpha$ versus the dimensionless body coordinate $x / b$ ( $b$ is an amplitude of the container vibration) is shown in Figure $7(\mathrm{a})$. One can see that the amplitude of the body oscillations is a little less than the amplitude of the container oscillations and the trajectory of the angle change with coordinate looks like a deformed ellipse. The direction of movement is indicated by an arrows. When the body moves to the right side, the angle $\alpha$ increases and the reverse movement is accompanied with a decrease in the angle of a slope. The trajectory of motion of the right edge of the body (solid symbols) and the left one (open ones) in the plane of the dimensionless parameters $x / h, d / \delta$, and $x / h, d / H$ is present in Figure $7(\mathrm{~b})$. Here the gap $d$ between the body edge and the container boundary is measured in the units of the thickness of the Stokes layer $\delta=\sqrt{2 \nu / \Omega}$ and the container height $H$ and the body coordinate $x$ in the units of the body thickness $h$. For clarity, the figure shows the entire working length of the cavity.

One can see that the body oscillates near the left end of the container touching the elastic bumper from time to time. The trajectory of each edge of the body has the shape of a deformed ellipse; arrows indicate the direction of movement. The dashed line shows the extreme right and left positions of the body. The distance at which the body is removed from the container boundary does not exceed the thickness of the Stokes layer, which is consistent with the previous results $[3,4]$.

The aluminum powder in the liquid visualizes the vortex structures excited in liquid by the oscillating body (Figure 7). In the above case, the Reynolds number responsible for the flow regime and calculated according to the formula $\operatorname{Re}=b \Omega h / \nu$ is about 1800. According to [10] at these Reynolds numbers, the vortex shedding from the edges of the oscillating body occurs.

4.2. The Body with $\rho=4$ 4.35. In case of lower relative density $\rho$, the steady average horizontal position of the body in suspended state is not found. In experiments, with increase of the vibration intensity at first only one of the body edges repulses. The angular body oscillations are no longer 


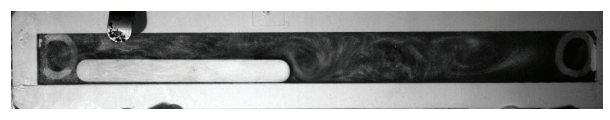

(a)

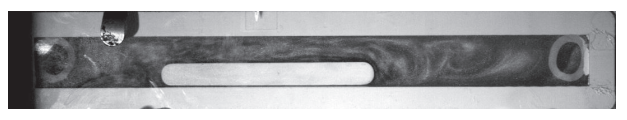

(b)

FIGURE 6: The body in the vibrating container in the extreme positions of the last, $b=33.4 \mathrm{~mm}, f=12.7 \mathrm{~Hz}, v=0.13 \mathrm{St}$.

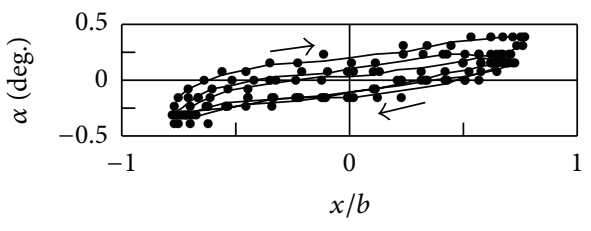

(a)

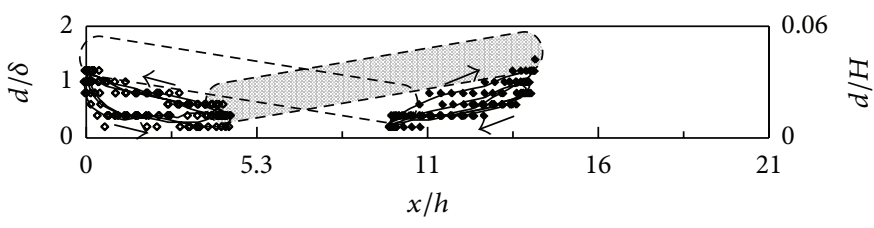

(b)

Figure 7: The change ot the body angle (a) and dimensionless gap (b) during the oscillations.

symmetric also. In this position, the body continuously moves along the bottom in the direction of a raised edge. Upon reaching the edge of the container, the body performs asymmetric vibrations near it.

The photos of the body when the container is in the extreme right (a) and the extreme left (b) positions are shown in Figure 8. The angle of the body tilt increases when the body approaches the container end.

Unlike the case $\rho=6.49$, the change of the angle sign during the angular body oscillations does not occur (Figure 9(a)). During the oscillations the body motion towards the container end (in this case the right displacement) is accompanied by an increase of $\alpha$ and the reverse body motion is accompanied by a decrease of $\alpha$. The relative amplitude $x / b$ of oscillations also increases with $\rho$. The trajectory of the body edges is not mirror-symmetric (Figure 9(b)). In the present case, the right edge of the body (solid symbols) is always at a distance from the container bottom, while the left edge (open symbols) is practically not detached from it. Note that the amplitude of the body oscillations is large compared to $h$.

4.3. The Body with $\rho=3.02$. The reduction of the relative density of the body to a value $\rho=3.02$ brings to the appearance of new subcritical regimes of the body behavior. The gap $d$ versus the frequency of container oscillations is shown in Figure 10. Slanted crosses in the graph denote the average gap between the right edge of the body and the container bottom and circles denote the average gap between the left edge and the bottom. The intervals indicate the deviation from the mean position during the period.

Consider the main regimes of the body. Point I corresponds to a stable suspension of the body at some distance from the bottom, just after the threshold transition of solid into a suspended state. The gap $d$ for the right and the left edges of the body is the same. The angular body oscillation is absent. With increase of the vibration frequency, the horizontal body position becomes unstable (points II). In this case, the right body edge repulses from the container boundary (crosses) while the left one (circles) stays near the bottom. This is similar to the behavior of earlier described body with $\rho=4.35$. With further increase of $f$ the angle of the body tilt rises. During the oscillations, the left body edge begins to break away from the bottom (points III). The tangential body motion along the container boundary occurs. During the body movement, the tilted body position remains (the right body edge is at a greater distance from the bottom than the left one). When reaching the left end of the container the change of the body orientation occurs, the left edge rises to the top and the right one goes to the bottom. After this transition, the solid starts motion in the opposite direction. With a slight further increase of $f$ the body goes away from the bottom at a large distance in the central part of the cavity (area IV). The gap $d$ rises with further intensification of the vibration and reaches a significant value. Let us consider each of the modes.

4.3.1. Point I. A qualitatively new quasi-steady suspended state is found in experiment with the body of relative density $\rho=3.02$. Just in the threshold, the body oscillates at very small distance from the container bottom without angular oscillations. The graph of the body and the container oscillations in this state is shown in Figure 11(a). The relative amplitude of the body oscillations $x / b \approx 0.4$ becomes smaller than the amplitudes of the previously described more dense bodies. The gap $d$ between the body and the container boundary (Figure 11(b)) is small, about a quarter of Stokes layer $\delta$. In this experiment, the body is near the right end of container but even in the proximity of the wall does not violate the horizontal position.

4.3.2. Points II and III. The increase of the oscillation frequency $f$ leads to the loss of sustained horizontal position. The body edge that is closer to the container end wall goes up while the opposite edge lays down on a bottom. The body makes oscillations along the container boundary and one of the body edges rests on it as in the case described above for the more dense body $(\rho=4.35)$.

With further increase of the vibration intensity the angle of the tilt increases to the limit value when the body edge substantially reaches the container top, while the other edge remains near the bottom. In this state, the body oscillations 


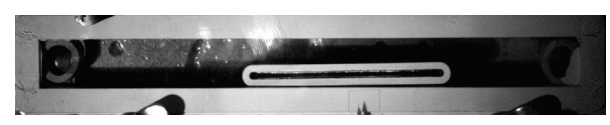

(a)

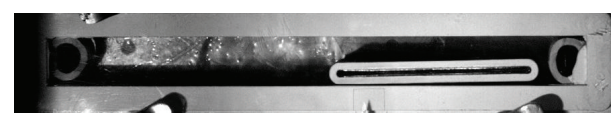

(b)

FIgURE 8: The body in the vibrating container in the extreme positions of the last, $b=32.6 \mathrm{~mm}, f=10.5 \mathrm{~Hz}, v=1.04 \mathrm{St}$.

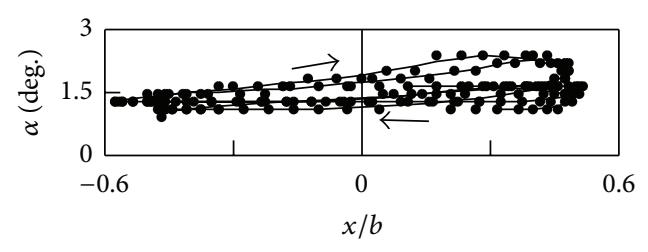

(a)

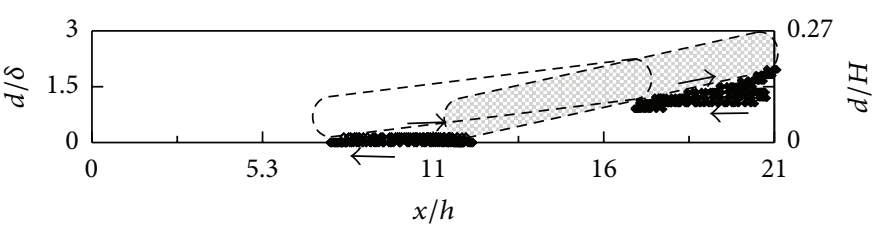

(b)

Figure 9: The change of the body angle (a) and the dimensionless gap (b) during the oscillations.

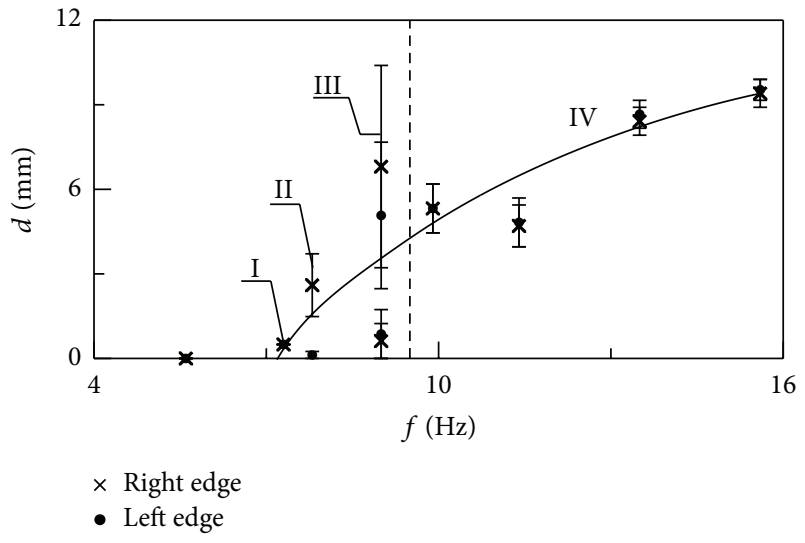

FIGURE 10: Regimes of the body behavior versus the vibration frequency, $b=34.6 \mathrm{~mm}, v=1.04 \mathrm{St}$.

generate the averaged tangential force, which causes the body to drift along the boundary of the cavity. When the body reached the opposite container end, the body changes its orientation and starts motion in the opposite direction. The drift direction of the body is determined by the edge, which is located at the container bottom. In this regime, the solid goes periodically from one cavity end to another. The process of changing the body orientation near the container end is presented in Figures 12 and 13. Figure 12 shows the change of the basic characteristics of the body oscillations over time. Figure 13 shows photographs of the container extreme positions and a trajectory of the body edges $(\mathrm{a}, \mathrm{b}, \mathrm{c})$ during the oscillation at different times. For convenience, in Figures 13(a)-13(c) the gap $d=10.8 \delta$ corresponds to the container height; the curves of the trajectories of body edges motion are duplicated for the lower and the upper edges of the body. The dashed line shows the extreme position of the oscillating body.

In Figure 12(a) the slope of curve of the body vibration relative to the container is clearly visible. That indicates the presence of the body drift along the container boundary; the oscillating body moves from right to left. During the oscillations in extreme right body position (moment of time $t_{1}$ ) the angle $\alpha$ is maximum (Figure 12(b)), right body edge is near the container top (Figure 12(c)), and left one is near the bottom (Figure 12(d)). In extreme left body position (moment $t_{2}$ ) the angle $\alpha$ significantly reduced and right body edge remains at some distance from the container boundary while the left one touches the bottom.

The angle $\alpha$ gradually decreases over time. At some moment (moments $t_{3}$ and $t_{4}$ ) the right body edge and left one are at the same height (Figures 12 and 13(a)). This condition resembles the equilibrium state of the body density $\rho=$ 6.49 committing longitudinal oscillations along the boundary and the angular oscillations of small amplitude. However, this body position is unstable. The right edge continues to fall with time (moment of time $t_{5}$ ) and the left one lifts to the container top (moment of time $t_{6}$ ) which leads to a change in the sign of the angle $\alpha$. After the change of the orientation in the container the body starts to drift towards the opposite container end wall, in which the transition process is repeated. Thus, the body performs strictly periodic motion from one end of the container to another. Despite the fact that the body drifts, the amplitude of oscillations $x / b$ remains approximately the same as in the stable stationary suspension state (Figure 14). Note that this regular regime of periodic drift of the body from one container end to another is observed in a very narrow frequency range.

4.3.3. Area $I V$. With further increase of $f$ the body reverts to a stable quasi-equilibrium state, this time at a considerable distance from the bottom (Figure 14). In this state, the body makes harmonic angular oscillation while being near the container end. The trajectories of the right and the left edges of the body are shown in Figure 14(c). During the oscillation, the body lifts up the right edge at the extreme right position and the left one at the extreme left position. Note that the body is in a middle height part of the container and oscillates at a distance of about $2 \delta \div 3 \delta$ both from the container bottom and from the container ceiling.

The relative amplitude of the body oscillations is similar compared to previous regimes (Figure 15). The amplitude of 


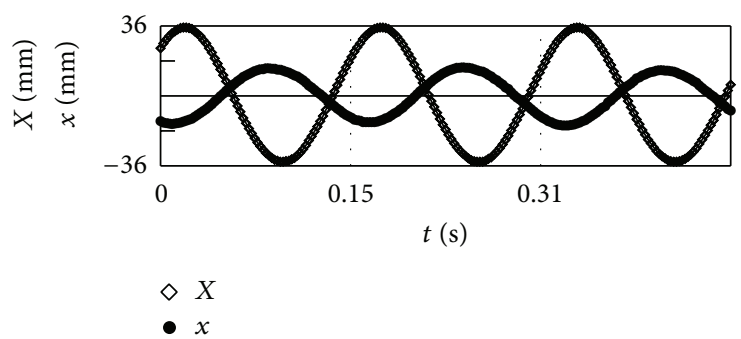

(a)

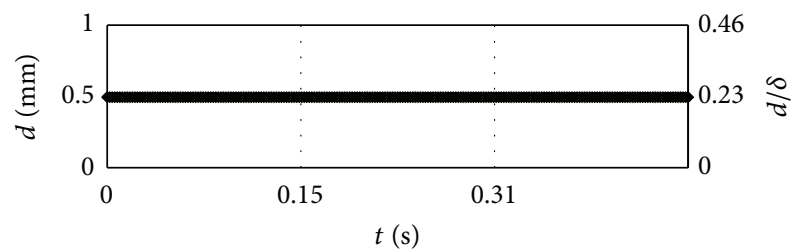

(b)

Figure 11: Characteristics of the body oscillations just after repulsion from the bottom, $f=7.3 \mathrm{~Hz}$.

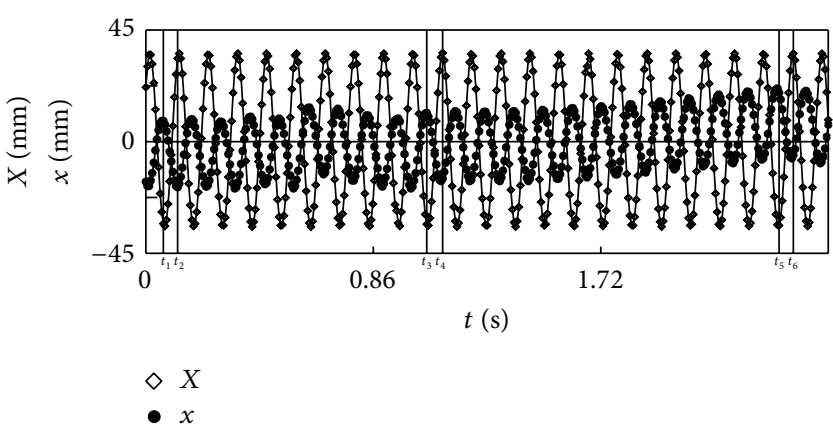

(a)

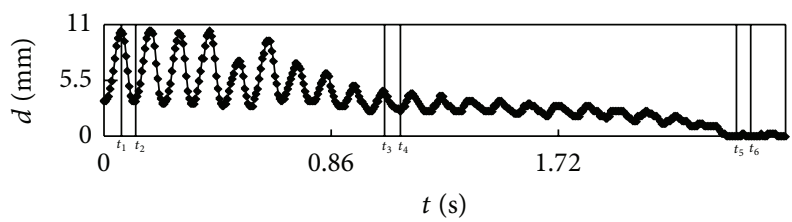

(c)

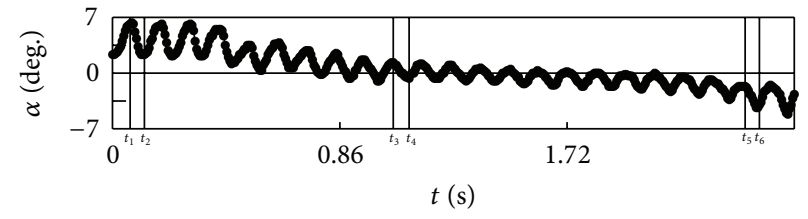

(b)

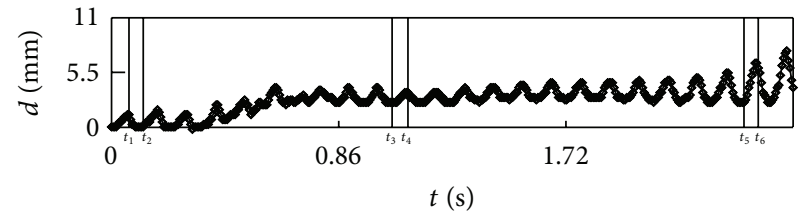

(d)

FIGURE 12: Characteristics of the body oscillation versus the time at point III (Figure 10), $f=9.0 \mathrm{~Hz}$.

angle body oscillations reaches the value of 1.2 degrees, which is several times higher than in case of the body $\rho=6.49$ oscillating near the bottom of the container.

In the subsequent increase of $f$ the body continues to move away from the bottom, until it occupies a stable position near the container ceiling (Figure 16). Near the upper boundary the body hangs at a distance from it and makes angular oscillations of small amplitude in complete analogy with the high-density bodies $(\rho=6.49)$ near the bottom. The body oscillates at distance comparable to the boundary layer thickness Stokes (Figure 16(c)).

Near the container ceiling the amplitude of angular oscillations $\alpha$ decreases (Figure 17). The body has some average angle of inclination due to the location near the left end of the container. The left edge of the body repels to a greater distance from the ceiling than the right one. Such a regime is similar to the oscillation behavior of the body $\rho=6.49$ near the lower boundary.

With monotonic decrease of the frequency of container vibration, all the regimes of the body oscillation and its position in the container repeat in a reverse order. The hysteresis in the regimes change was not found.

\section{Analysis of the Experimental Results}

In [9] the lift force acting on the flat body with relative thickness $\xi=h_{S} /\left(H-h_{S}\right)$ has been calculated. The body makes longitudinal oscillations with vibration frequency $\Omega$ near the flat boundary of the channel thickness $H$. The lift force acting per unit body surface and repulsing the body from the wall is given by

$$
\overline{\Delta p}=\frac{1}{4} \frac{(1+\xi)^{2}(\rho-1)^{2}}{(\rho+\xi)^{2}} \rho_{L} b^{2} \Omega^{2} .
$$

The lift force is generated by the oscillation of the body relative to the container and a piston fluid oscillation between the body and the opposite wall of the container. The amplitude of body oscillations is assumed small and the frequency high, and the fluid oscillates as inviscid. An oscillating force of inertia associated with the container longitudinal translational vibrations causes the body oscillation in the container. The body and the cavity amplitude are related by the formula $A / b=(\rho-1) /(\rho+\xi)$. 


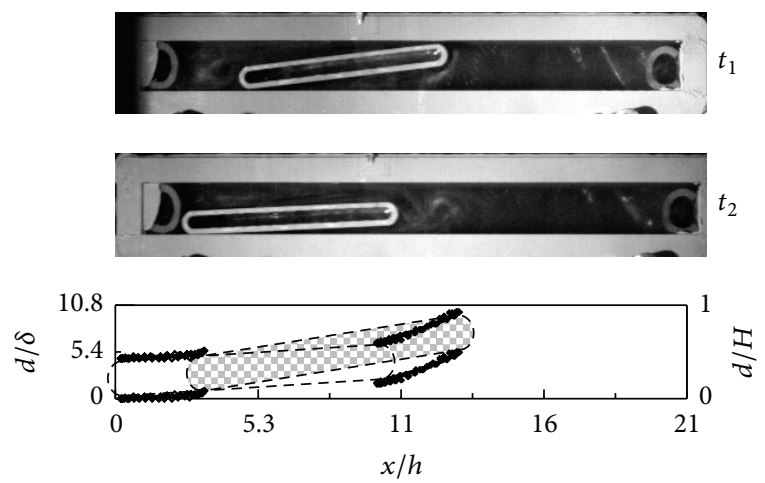

(a)
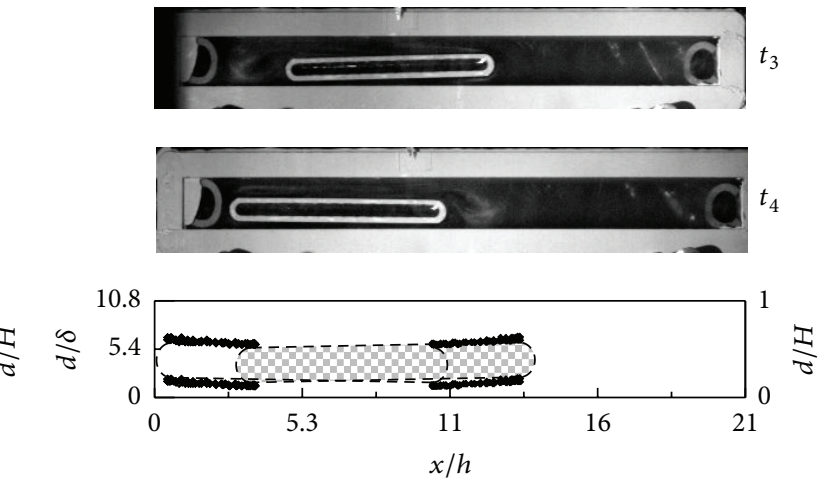

(b)
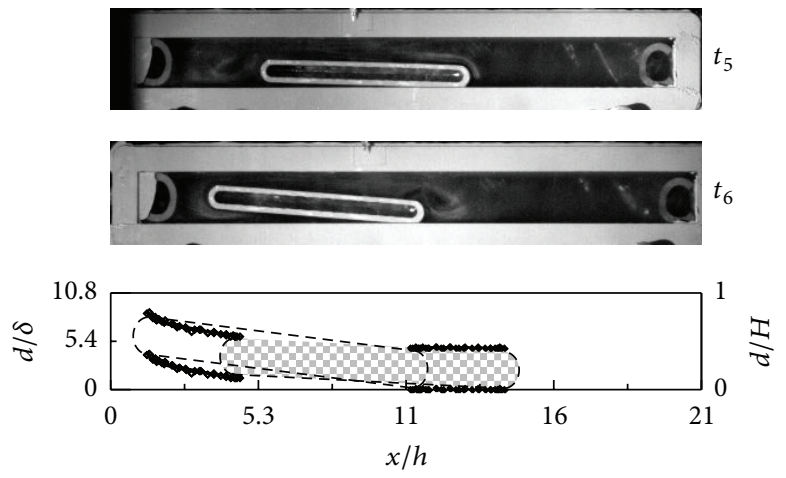

(c)

FiguRE 13: The oscillating body motion trajectory at different times during transition process near the left container end; time moments $\left(t_{1}-t_{6}\right)$ are marked in Figure 12.

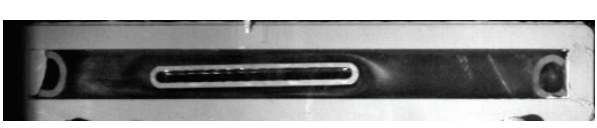

(a)

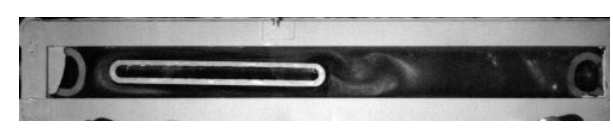

(b)

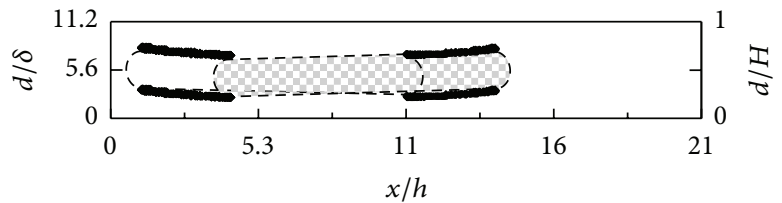

(c)

FIgURE 14: The body in the extreme position of the container during vibration ((a): left, (b): right) and the oscillating body motion trajectory (c) during the oscillation, $f=9.9 \mathrm{~Hz}$.

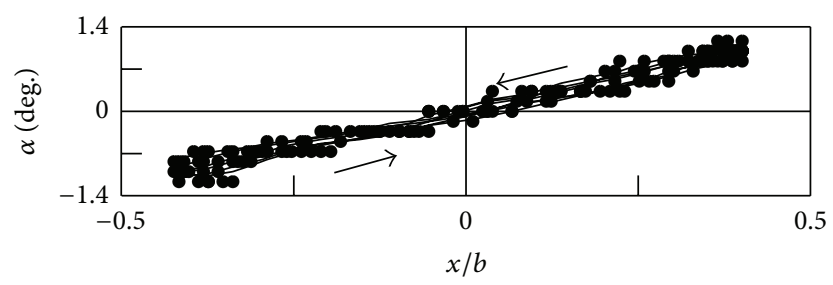

FIGURE 15: The angle of the body tilt variation during the oscillation. 


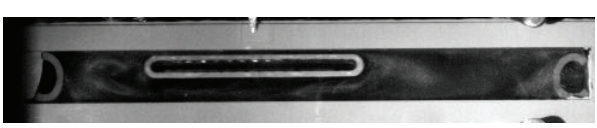

(a)

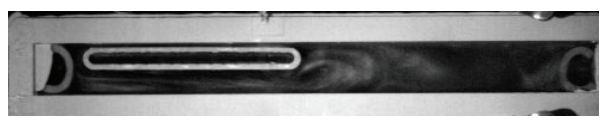

(b)

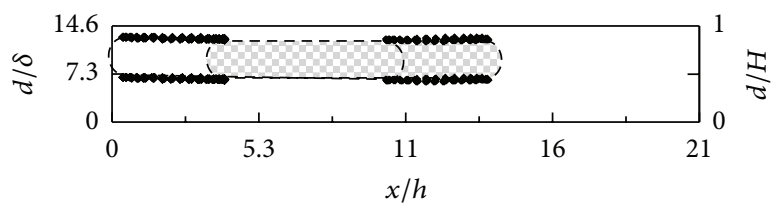

(c)

Figure 16: Flat solid position in the container during vibration ((a): left, (b): right) and the trajectory of oscillating body motion (c) during the period, $f=15.6 \mathrm{~Hz}$.

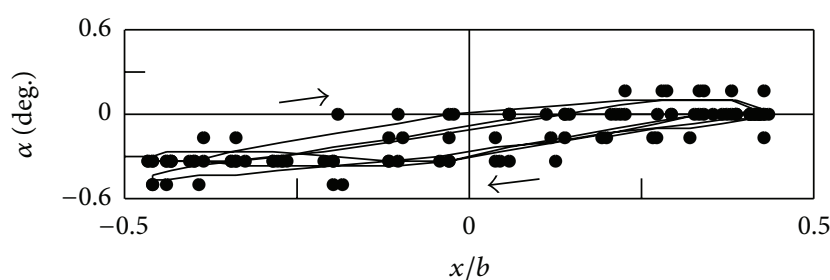

FIGURE 17: The angle of the body tilt during the oscillation near the cavity ceiling.

In experiments in the gravity field, this force can be measured by the suspension method, when the repulsion lift force is balanced by the body weight, and the body goes into a suspended state. Conditions of the body balance at a short distance from the solid boundary follow from the relation

$$
\frac{1}{4} \frac{(1+\xi)^{2}(\rho-1)^{2}}{(\rho+\xi)^{2}} \rho_{L} b^{2} \Omega^{2}=\left|\rho_{S}-\rho_{L}\right| h_{S} g
$$

To compare the results of experiments performed with the bodies of different density, it is convenient to introduce the dimensionless lift force in the next form:

$$
F_{L}=\frac{h_{S} g(\rho+\xi)^{2}}{b^{2} \Omega^{2}(\rho-1)}
$$

In this case, the critical value of $F_{L}^{*}$ when the body is suspended (in the approximation of small amplitude and high frequency vibration) is given by

$$
F_{L}^{*}=\frac{(\xi+1)^{2}}{4}
$$

Under our experimental conditions, $\xi=0.77$, the critical value of the lift force when the body holds at some distance from the boundary is $F_{L}^{*}=0.78$. The dependence of vibrational lift force calculated by formula (3) on the dimensionless amplitude of body oscillations $A / h$ is shown in Figure 18. Unlike the theory, under the conditions of our experiments the body has a finite length and oscillates with an amplitude considerably more than its own thickness. This type of oscillations is characterized by separated flow around the body and the vortex shedding. As can be seen from the graph the critical value of lift force is different from the theory (dashed line). At the same time, all the results obtained in experiments with the bodies of different density in liquids with different viscosity are in satisfactory agreement with each other on the plane of these parameters. With the increase of $A / h$ the vibrational lift force decreases monotonically.

For comparison, the graph shows the experimental points obtained in the preliminary experiments [9] with a different solid body ( $\rho \sim 2, \xi=0.79$ ) with relatively sharp edges. These results qualitatively confirm the existence of lift force but are in poor agreement with the present data obtained with a heavy body with a smooth rounded edges. Thus, the experimental points divide into two groups. The points corresponding to the experiments for low-viscosity liquids are located above the points obtained in experiments with heavy bodies. It should be noted that in these experiments the body in suspended state makes irregular chaotic oscillations caused by vortex shedding from the sharp body edges. In the experiments with fluids of high viscosity, the body makes regular oscillations, while the experimental points are lower than for the more dense bodies. In addition to irregularly shaped body, which had a sharp edge, this can be explained by the fact that the experiments [9] were performed in a container of shorter relative length.

Summarizing the experimental findings, we see that the behavior of heavy bodies, which oscillate with large amplitude, is different from the vibration inherent in the theoretical model. In the experiment, there are slight angular body oscillations (Figure 6). With decreasing the relative body density, the average horizontal position of the suspended body becomes unstable. One of the body edges rises above the container bottom, the other remains near it, and at the same time the body makes low amplitude angular oscillations (Figure 8). In experiment, we find the mean body interaction with the side ends of the container. Thus, the body position in the container center is unstable; the bodies are attracted either to the right container end or to the left one. This phenomenon lies within an averaged force excited by oscillating potential flow around the body [2]. According to our experiments, the interaction with the container end walls results in the rise of the body edge located near the wall (Figure 9). With decrease 


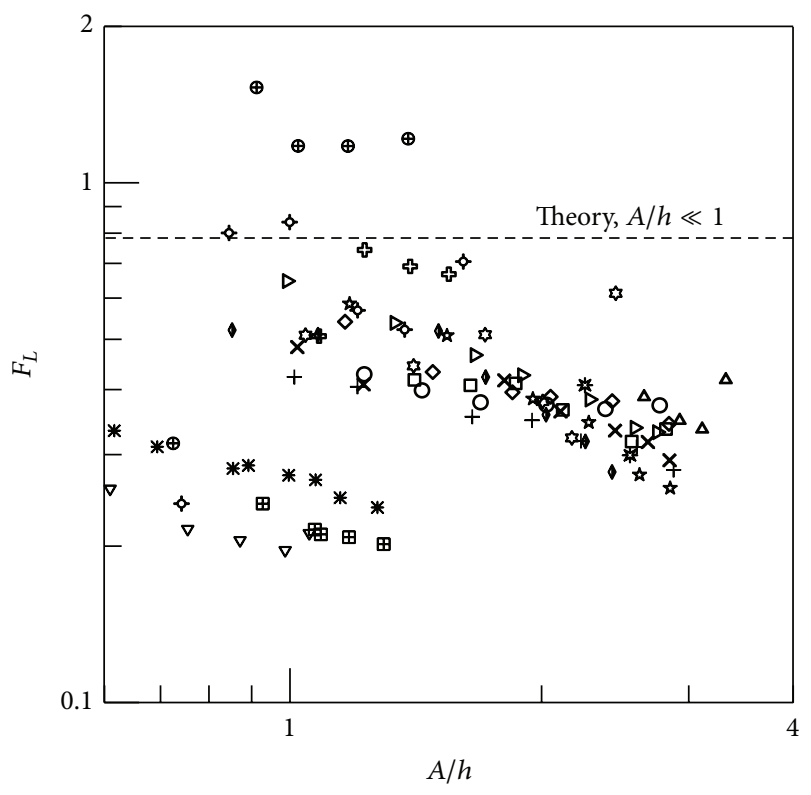

\begin{tabular}{|c|c|c|c|c|}
\hline \multicolumn{5}{|l|}{ Aluminum } \\
\hline$v, \mathrm{St}$ & & $v, \mathrm{St}$ & & \\
\hline$\Leftrightarrow 0.26$ & $\nabla$ & 1.29 & & \\
\hline \& 0.14 & 田 & 0.59 & & \\
\hline $\begin{array}{l}\oplus 0.05 \\
\rho=2.0-2.1\end{array}$ & * & 0.42 & & \\
\hline \multicolumn{5}{|l|}{ Bronze } \\
\hline$v, \mathrm{St}$ & & $v, \mathrm{St}$ & & $v, \mathrm{St}$ \\
\hline$\square 1.21$ & $x$ & 1.06 & $\Delta$ & 1.04 \\
\hline ○ 0.42 & + & 1.05 & as & 0.32 \\
\hline$\diamond 0.13$ & ڤ્ & 0.31 & 沶 & 0.13 \\
\hline$\Delta 0.12$ & $\triangleright$ & 0.11 & & $=3.0-3.1$ \\
\hline$\rho=6.5-6.3$ & $\rho$ & $=4.2-4.4$ & & \\
\hline
\end{tabular}

FIGURE 18: The dependence of the lift force on the amplitude of body oscillation.

of the relative body density, the height of rising increases. Qualitatively new is the regime when the body edge lifts to the container ceiling (Figure 13). In this case, a new phenomenon, the emergence of a tangential lift force driving the body along the container boundary, manifests itself. Keeping its tilted position, the body moves to the opposite container end where the angle of the slope changes. This process has a selfoscillating character.

One more unexpected phenomenon is the body repulsion from the lower boundary, when it occupies a quasi-stationary stable position in the bulk of the cavity and then is attracted to the upper boundary (area IV on Figure 10). In this state, the body also experiences a weak pull to the side edges of the cavity. When the body reaches the container end and bumps it, it takes a position in the central part of the container length and then again slowly returns to the container end. The observed phenomenon of the gradual removal of the body from the bottom with an increase of vibration intensity and the transition to a stable state near the ceiling of the oscillating cavity (area IV on Figure 10) does not fit in the developed theory and is of considerable interest.
It is known that a similar behavior of the body is possible in case of the nontranslational oscillations of the container [11]. However, in our experiments, this phenomenon cannot occur because the translational character of the container oscillations is strictly controlled. One can suppose that the observed phenomenon could be determined by the relatively small thickness of the channel; it is observed at moderate dimensionless frequency when the channel height and the body thickness are comparable with the thickness of the Stokes layer. The observed effect is not clear and needs further investigation.

\section{Conclusion}

The experimental study of the behavior of a heavy body in the form of parallelepiped with rounded edges in the container of rectangular cross section, which makes the translational horizontal oscillations, is conducted. It is found that the vibrations result in a generation of strong lift force of repulsion; the body of which density is several times greater than the liquid density lifts up and goes to quasi-steady suspended state at some distance from the container bottom.

It is shown that a threshold of vibrational suspension of the heavy body is in agreement with the theoretical data in the limiting case of small amplitude of body oscillations. The lift force reduces with increasing the dimensionless amplitude of body oscillations in the area of large dimensionless amplitudes.

A new phenomenon is found in the case of moderate $h / \delta$ when the body size is comparable to the thickness of the Stokes layer; it consists in progressive displacement of the point of equilibrium suspended state of the body from the lower boundary to the upper one with increasing the intensity of vibration.

\section{Competing Interests}

The authors declare that there is no conflict of interests regarding the publication of this paper.

\section{Acknowledgments}

The work is supported by Grant 14-11-00476 of the Russian Science Foundation.

\section{References}

[1] V. L. Sennitskii, "Motion of a sphere in a vibrating liquid in the presence of a wall," Journal of Applied Mechanics and Technical Physics, vol. 40, no. 4, pp. 662-668, 1999.

[2] D. V. Lyubimov, T. P. Lyubimova, and A. A. Cherepanov, "On a motion of solid body in a vibrating fluid," in Convective Flows, E. M. Zhukhovitsky, Ed., pp. 61-70, 1987.

[3] A. A. Ivanova, V. G. Kozlov, and A. F. Kuzaev, "Vibrational lift force acting on a body in a fluid near a solid surface," Doklady Physics, vol. 50, no. 6, pp. 311-314, 2005.

[4] A. A. Ivanova, V. G. Kozlov, and V. D. Shchipitsyn, "Lift force acting on a cylindrical body in a fluid near the boundary of a cavity performing translational vibrations," Journal of Applied 
Mechanics and Technical Physics, vol. 55, no. 5, pp. 773-780, 2014.

[5] S. Hassan, T. P. Lyubimova, D. V. Lyubimov, and M. Kawaji, "Effects of vibrations on particle motion near a wall: existence of attraction force," International Journal of Multiphase Flow, vol. 32, no. 9, pp. 1037-1054, 2006.

[6] M. Saadatmand, M. Kawaji, and H. H. Hu, "Vibration-induced attraction of a particle towards a wall in microgravity-the mechanism of attraction force," Microgravity Science and Technology, vol. 24, no. 1, pp. 53-64, 2012.

[7] M. Saadatmand and M. Kawaji, "Mechanism of vibrationinduced repulsion force on a particle in a viscous fluid cell," Physical Review E-Statistical, Nonlinear, and Soft Matter Physics, vol. 88, no. 2, Article ID 023019, 2013.

[8] M. Saadatmand and M. Kawaji, "Parametric study of the vibration-induced repulsion or attraction force on a particle in a viscous fluid cell," Physical Review E, vol. 89, no. 4, Article ID 43009, 2014.

[9] V. Kozlov and O. Vlasova, "The repulsion of flat body from the wall of vibrating container filled with liquid," Microgravity Science and Technology, vol. 27, no. 4, pp. 297-303, 2015.

[10] B. M. Sumer and J. Fredsøe, Hydrodynamics around Cylindrical Structures, vol. 12, World Scientific, 1997.

[11] V. G. Kozlov, "Solid-body dynamics in cavity with liquid under high-frequency rotational vibration," Europhysics Letters, vol. 36, no. 9, pp. 651-656, 1996. 


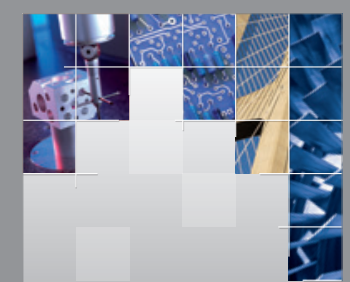

\section{Enfincering}
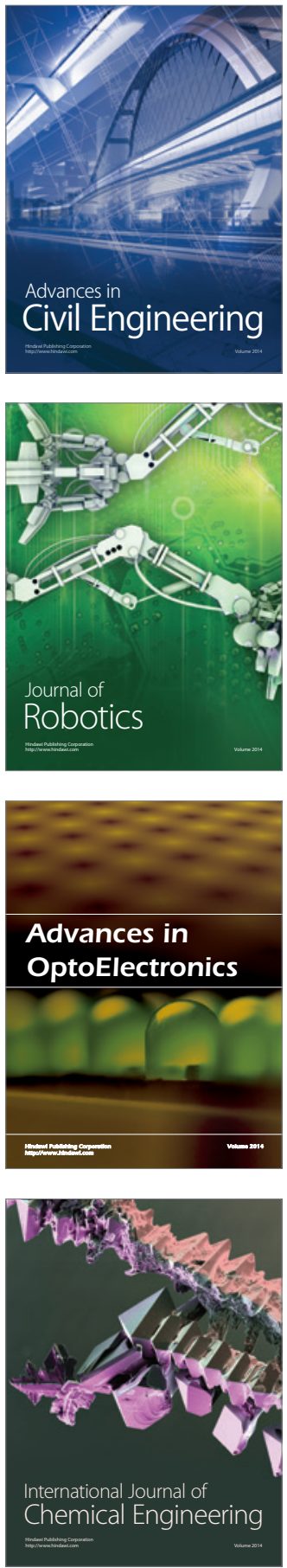

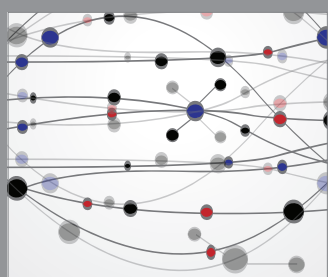

The Scientific World Journal

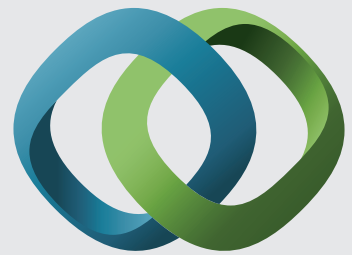

\section{Hindawi}

Submit your manuscripts at

http://www.hindawi.com
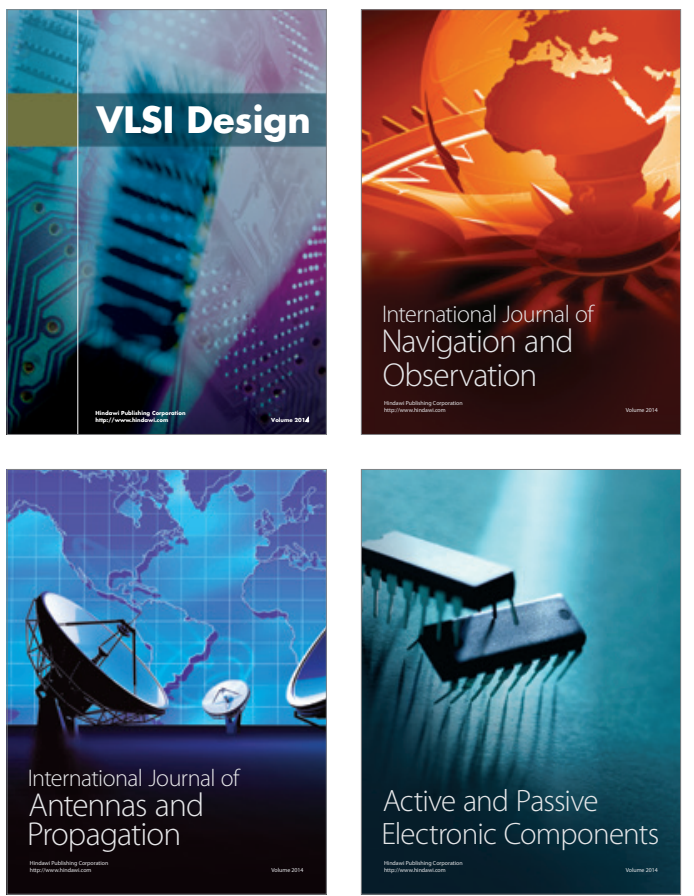
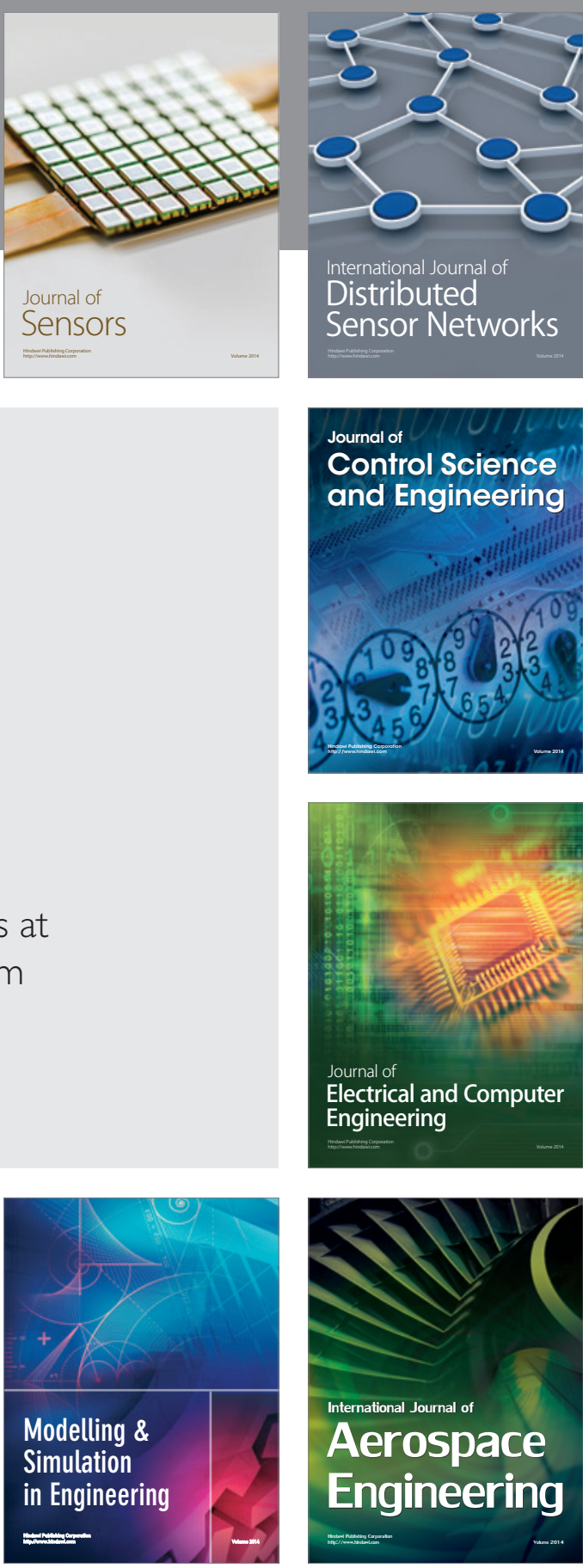

International Journal of

Distributed

Sensor Networks

Journal of

Control Science

and Engineering
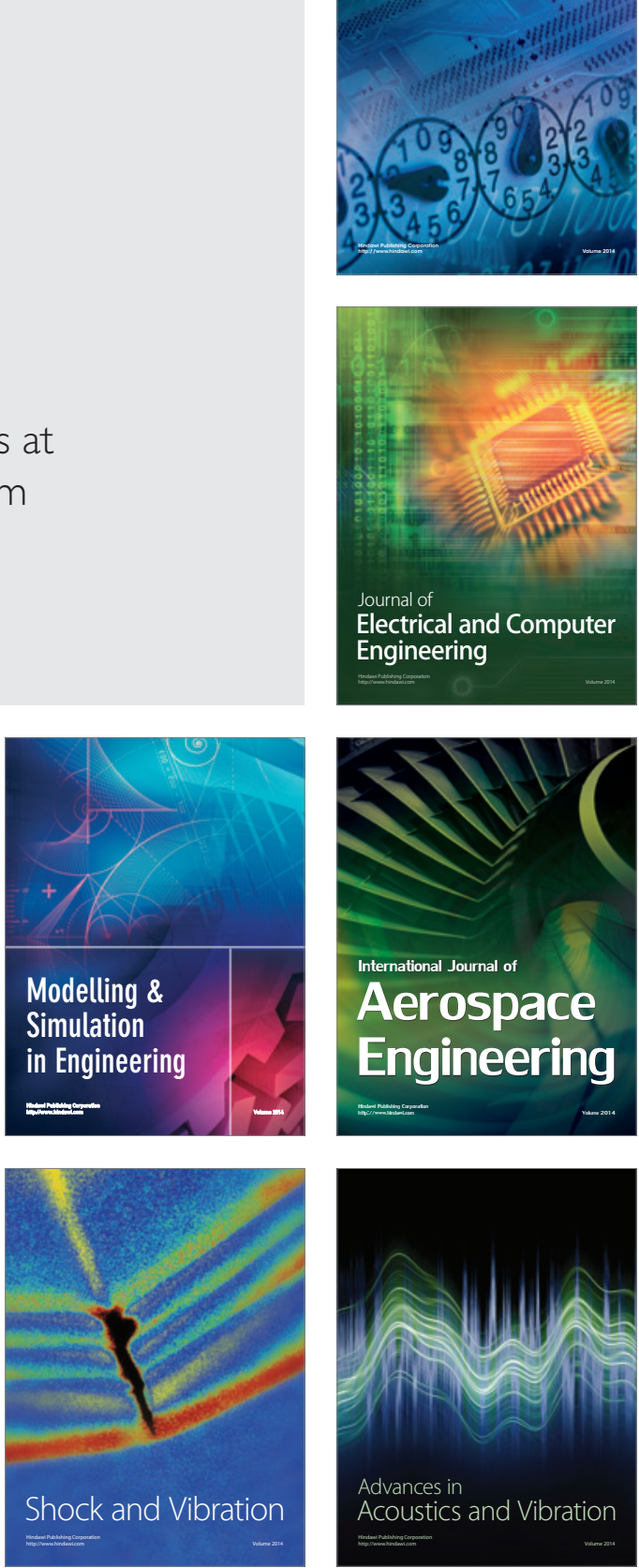